\title{
Urinary $\beta_{2}$-Microglobulin Testing in Pediatric Uveitis: A Case Report of a 9-Year-Old Boy with Renal and Ocular Sarcoidosis
}

\author{
Kathryn L. Pepple ${ }^{a} \quad$ Deborah L. Lam ${ }^{a} \quad$ Laura S. Finn ${ }^{\text {b, d }}$ \\ Russell Van Gelder ${ }^{a-c}$ \\ Departments of ${ }^{\mathrm{a} O p h t h a l m o l o g y,}{ }^{\mathrm{b}}$ Pathology, and ${ }^{\mathrm{C}}$ Biological Structure, University of \\ Washington Medical School, and dDepartment of Laboratories, Seattle Children's Hospital, \\ Seattle, Wash., USA
}

\section{Key Words}

Tubulointerstitial nephritis and uveitis syndrome $\cdot \beta_{2}$-Microglobulin · Sarcoidosis · Uveitis · Juvenile idiopathic arthritis

\begin{abstract}
We present here a case of a 9-year-old boy with bilateral anterior uveitis and an extremely elevated urinary $\beta_{2}$-microglobulin level $(25,400 \mu \mathrm{g} / \mathrm{l})$. The normal range for urinary excretion of $\beta_{2}$-microglobulin is $0-300 \mu \mathrm{g} / \mathrm{l}$. In patients with tubulointerstitial nephritis and uveitis syndrome (TINU), elevations typically range from 1,260 to 5,160 $\mu \mathrm{g} / \mathrm{l}$. Renal biopsy was pursued, and significant granulomatous interstitial nephritis consistent with sarcoidosis was identified. Systemic immune modulation was required for control of ocular inflammation. This case highlights the importance of urinary $\beta_{2}$-microglobulin testing in the pediatric patient uveitis population, and additionally the need to pursue kidney biopsy in the presence of extreme elevations in urinary $\beta_{2}$-microglobulin to differentiate between TINU and sarcoidosis.
\end{abstract}

(C) 2015 S. Karger AG, Basel

\section{Introduction}

The most common cause of bilateral noninfectious uveitis in the pediatric population is juvenile idiopathic arthritis (JIA)-associated uveitis [1]. However, due to the high incidence of tubulointerstitial nephritis and uveitis syndrome (TINU) in the pediatric population, the

KARGER 125/s $\quad \begin{aligned} & \text { Kathryn L. Pepple, MD, PhD } \\ & \text { Department of Ophthalmology } \\ & \text { University of Washington Medical School } \\ & \text { Seattle, WA } 98104-2499 \text { (USA) } \\ & \text { E-Mail kpepple@ uw.edu }\end{aligned}$


Pepple et al.: Urinary $\beta_{2}$-Microglobulin Testing in Pediatric Uveitis: A Case Report of a 9-Year-Old Boy with Renal and Ocular Sarcoidosis

complete workup must also include testing for increased urinary $\beta_{2}$-microglobulin levels [24]. We report a patient with bilateral anterior uveitis with a remarkable elevation in urinary $\beta_{2}$-microglobulin levels (five times the levels normally seen with TINU), and a kidney biopsy that revealed sarcoidosis as the underlying etiology. Sarcoidosis is uncommon in children, with an annual incidence of 2-3 cases per million [5], and is responsible for less than $3 \%$ of pediatric uveitis cases [6-8]. This case suggests that in the presence of extremely elevated urinary $\beta_{2}$-microglobulin levels, sarcoidosis rather than TINU is the underlying etiology.

\section{Case Presentation}

A 9-year-old Caucasian male was referred to our Department for evaluation and management of a 4-month history of chronic bilateral anterior uveitis. His initial symptoms included recurrent right eye redness, pain, and photophobia, in conjunction with right leg pain. At his initial ophthalmology exam, cell and flare were noted in both eyes, and he was diagnosed with rheumatoid factor-negative, antinuclear antibody-positive JIA and started on difluprednate drops in both eyes. After a month of topical therapy, the anterior chamber inflammation in both eyes failed to resolve.

At presentation to the uveitis service, he complained of photophobia and blurry vision, but without eye redness. He was using prednisolone acetate 1\% drops four times a day in the right eye, difluprednate drops every $2 \mathrm{~h}$ in the left eye, and homatropine and timolol/brimonidine combination drops twice daily in both eyes.

His past medical history included exercise-induced asthma. His review of symptoms was positive for fatigue and right knee and ankle pain associated with sporting activities without joint swelling, but negative for weight loss, fevers, cough, and rash.

On examination, the patient was a healthy-appearing male child. Vision without correction in the right eye was 20/125 improving to 20/80-2 with pinhole, and 20/70 in the left eye improving to $20 / 40+2$. His intraocular pressures were 15 and 14 . There was no relative afferent pupillary defect. Motility and confrontational visual fields were full in both eyes.

The eyes were quiet, and corneas were clear without keratic precipitates. The anterior chambers were formed and deep with 2+ cell and 1+ flare by the Standardization of Uveitis Nomenclature criteria in both eyes. Posterior synechiae were noted in the right eye. No iris nodules were noted, and the lenses were clear. Anterior vitreous cell and subfoveal fluid was noted in the right eye. Small punched out chorioretinal lesions were noted in the inferior periphery of both eyes. No vitritis, retinitis, vasculitis or optic nerve edema was present in either eye.

Laboratory evaluation tests revealed a normal chest X-ray, slightly elevated serum creatinine, a urinalysis positive for $1+$ urinary proteins, and a markedly elevated urine $\beta_{2^{-}}$ microglobulin at 25,400 $\mu \mathrm{g} / \mathrm{l}$ (normal 0-300 $\mu \mathrm{g} / \mathrm{l}$, TINU range 1,260-5,160 $\mu \mathrm{g} / \mathrm{l}$ [2]). A renal biopsy was then performed demonstrating granulomatous interstitial nephritis and patchy interstitial fibrosis (fig. 1). Biopsies from patients with TINU (fig. 2) infrequently demonstrate granulomas or multinucleated foreign body-type giant cells that can be associated with damaged tubules. However, the finding of several cortical noncaseating granulomas in this case was more consistent with sarcoidosis [9-11]. After biopsy, topical therapy was supplemented with $40 \mathrm{mg}$ oral prednisone daily. Serum creatinine and urinary $\beta_{2}$ microglobulin improved rapidly. However, uveitis control required parenteral MTX (25 $\mathrm{mg} /$ week) and Humira (40 mg/every other week). On this regimen, inflammation was quiescent, and vision stabilized at 20/60 after cataract surgery in the right eye and 20/25 with a posterior subcapsular cataract in the left eye. 
Pepple et al.: Urinary $\beta_{2}$-Microglobulin Testing in Pediatric Uveitis: A Case Report of a 9-Year-Old Boy with Renal and Ocular Sarcoidosis

\section{Discussion}

We present this case to illustrate two important points in the diagnosis and management of pediatric uveitis. First, not all uveitis in children is JIA-associated uveitis. Sarcoidosis and TINU should also be considered, particularly in any child presenting with a symptomatic bilateral anterior uveitis. The initial screening should include a chest X-ray, urinalysis, urinary $\beta_{2}$-microglobulin, and serum creatinine.

TINU is a rare syndrome characterized by anterior uveitis in combination with acute interstitial nephritis (AIN). TINU comprises around 1-2\% of cases seen in subspecialty practices, but may represent up to a third of cases of bilateral acute-onset anterior uveitis in patients younger than $20[2,4,12,13]$. Patients will present with a symptomatic nongranulomatous anterior uveitis that is typically bilateral on presentation, or becomes bilateral within a few months [12]. Systemic symptoms of nephritis such as fever, weight loss, and fatigue are common. Uveitis presents within a month of development of renal manifestations in $80 \%$ of patients. Recurrent or chronic disease develops in less than half of patients [12].

When TINU is suspected, referral to a nephrologist for further evaluation and possible kidney biopsy is recommended. Ultimately, TINU syndrome is a diagnosis of exclusion made in patients with both an AIN and uveitis, but it also requires a negative evaluation for other possible systemic causes. In this case, the biopsy performed for the evaluation of TINU demonstrated granulomatous inflammation that raised the consideration of sarcoidosis. The criteria for the diagnosis of TINU proposed by Mandeville et al. [12] include the demonstration of an AIN by appropriate clinical presentation or by histopathology. The most common pathological feature of AIN is the presence of a mixed inflammatory cell infiltrate within the kidney interstitium [14]. While bone marrow granulomas were described in the first patients with TINU, and interstitial granulomas can be seen in some patients with AIN from a drug hypersensitivity reaction, this is not a typical feature of AIN. However, this overlap in presentation can make differentiating between a primary AIN and another cause of granulomatous interstitial nephritis such as sarcoidosis difficult. It also raises the interesting possibility that in some cases, the diagnosis of TINU and sarcoidosis represent a disease spectrum rather than distinct clinical entities. In this case, with elimination of common etiologies of granulomatous interstitial nephritis such as drug (hypersensitivity) reaction, foreign material or infection, the burden of granulomatous disease on histology was used to determine that sarcoidosis was the most likely cause of the patient's ocular and renal disease.

Sarcoidosis is a chronic granulomatous disease of unknown etiology that is characterized by the presence of noncaseating granulomas [15]. Sarcoidosis is uncommon in children, with one large study from Denmark reporting an annual incidence of 2-3 cases per million [5], and is responsible for $0.9-3 \%$ of pediatric uveitis cases [1, 6-8]. Sarcoidosis in children can manifest in two distinct manners. The early onset form presents in young children, typically less than 5 years of age, and is characterized by the triad of rash, uveitis, and arthritis. Older children usually present with a multisystem disease similar to that seen in adults including hilar lymphadenopathy, uveitis, and skin manifestations $[6,16]$. In older children, the most common eye manifestations are also similar to those found in adults including anterior uveitis, multifocal choroiditis, and periphlebitis [6]. However, the diagnosis can be difficult to make without a biopsy due to the lower frequency of hilar lymphadenopathy on chest X-ray, and the physiological elevation of the angiotensin-converting enzyme in children [1]. Corticosteroids are the first line of therapy for pediatric patients with ocular sarcoidosis. However, timely initiation of steroid-sparing therapy with methotrexate is advised in cases requiring systemic therapy to prevent the adverse effects of corticosteroids on growth and metabolism. 
Pepple et al.: Urinary $\beta_{2}$-Microglobulin Testing in Pediatric Uveitis: A Case Report of a 9-Year-Old Boy with Renal and Ocular Sarcoidosis

This case highlights the importance of going beyond JIA in the evaluation of an acute bilateral anterior uveitis, particularly when the presentation has features such as pain, redness, and photophobia. Furthermore, this case highlights the importance of a complete evaluation to rule out sarcoidosis as an underlying cause of the TINU syndrome, particularly when very elevated urinary $\beta_{2}$-microglobulin is identified.

In summary, TINU and sarcoidosis are underrecognized causes of pediatric uveitis, and can be overlooked if not considered in the differential diagnosis.

\section{Acknowledgements}

K.L.P. is supported by NIH award K08EY023998. R.V.G. is supported by the BurroughsWellcome Translational Scientist Award, an unrestricted award from Research to Prevent Blindness, and by NIH P30 EY001730.

\section{References}

1 Cunningham ET Jr: Uveitis in children. Ocul Immunol Inflamm 2000;8:251-261.

-2 Hettinga YM, Scheerlinck LM, Lilien MR, Rothova A, de Boer JH: The value of measuring urinary $\beta 2$ microglobulin and serum creatinine for detecting tubulointerstitial nephritis and uveitis syndrome in young patients with uveitis. JAMA Ophthalmol 2015;133:140-145.

-3 Kase S, Kitaichi N, Namba K, Miyazaki A, Yoshida K, Ishikura K, Ikeda M, Nakashima T, Ohno S: Elevation of serum Krebs von den Lunge-6 levels in patients with tubulointerstitial nephritis and uveitis syndrome. Am J Kidney Dis 2006;48:935-941.

4 Mackensen F, Smith JR, Rosenbaum JT: Enhanced recognition, treatment, and prognosis of tubulointerstitial nephritis and uveitis syndrome. Ophthalmology 2007;114:995-999.

5 Hoffmann AL, Milman N, Byg KE: Childhood sarcoidosis in Denmark 1979-1994: incidence, clinical features and laboratory results at presentation in 48 children. Acta Paediatr 2004;93:30-36.

6 Choi DE, Birnbaum AD, Oh F, Tessler HH, Goldstein DA: Pediatric uveitis secondary to probable, presumed, and biopsy-proven sarcoidosis. J Pediatr Ophthalmol Strabismus 2011;48:157-162.

7 Kump LI, Cervantes-Castaneda RA, Androudi SN, Foster CS: Analysis of pediatric uveitis cases at a tertiary referral center. Ophthalmology 2005;112:1287-1292.

8 BenEzra D, Cohen E, Maftzir G: Uveitis in children and adolescents. Br J Ophthalmol 2005;89:444-448.

19 Berliner AR, Haas M, Choi MJ: Sarcoidosis: the nephrologist's perspective. Am J Kidney Dis 2006;48:856870 .

10 Bijol V, Mendez GP, Nose V, Rennke HG: Granulomatous interstitial nephritis: a clinicopathologic study of 46 cases from a single institution. Int J Surg Pathol 2006;14:57-63.

11 Joss N, Morris S, Young B, Geddes C: Granulomatous interstitial nephritis. Clin J Am Soc Nephrol 2007;2:222-230.

12 Mandeville JT, Levinson RD, Holland GN: The tubulointerstitial nephritis and uveitis syndrome. Surv Ophthalmol 2001;46:195-208.

13 Mackensen F, Billing H: Tubulointerstitial nephritis and uveitis syndrome. Curr Opin Ophthalmol 2009;20:525-531.

Michel DM, Kelly CJ: Acute interstitial nephritis. J Am Soc Nephrol 1998;9:506-515.

Culver DA: Sarcoidosis. Immunol Allergy Clin North Am 2012;32:487-511.

Shetty AK, Gedalia A: Childhood sarcoidosis: a rare but fascinating disorder. Pediatr Rheumatol Online J 2008;6:16. 


\section{Case Reports in \\ Ophthalmology}

\begin{tabular}{l|l}
\hline \multicolumn{2}{l}{ Case Rep Ophthalmol 2015;6:101-105 } \\
\hline DOI: 10.1159/000381092 & $\begin{array}{l}\text { ○ 2015 S. Karger AG, Basel } \\
\text { www.karger.com/cop }\end{array}$ \\
\hline
\end{tabular}

Pepple et al.: Urinary $\beta_{2}$-Microglobulin Testing in Pediatric Uveitis: A Case Report of a 9-Year-Old Boy with Renal and Ocular Sarcoidosis

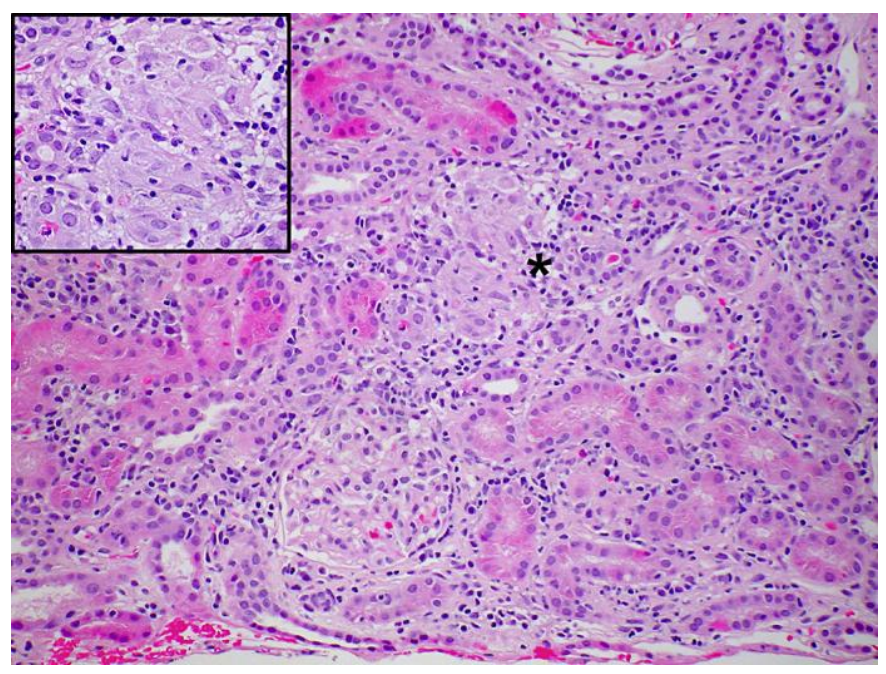

Fig. 1. Sarcoidosis. Inflammation comprising noncaseating epithelioid granulomas (asterisk, inset) and lymphocytes expands the tubulointerstitial compartment of the kidney and spares the glomerulus (midlower).

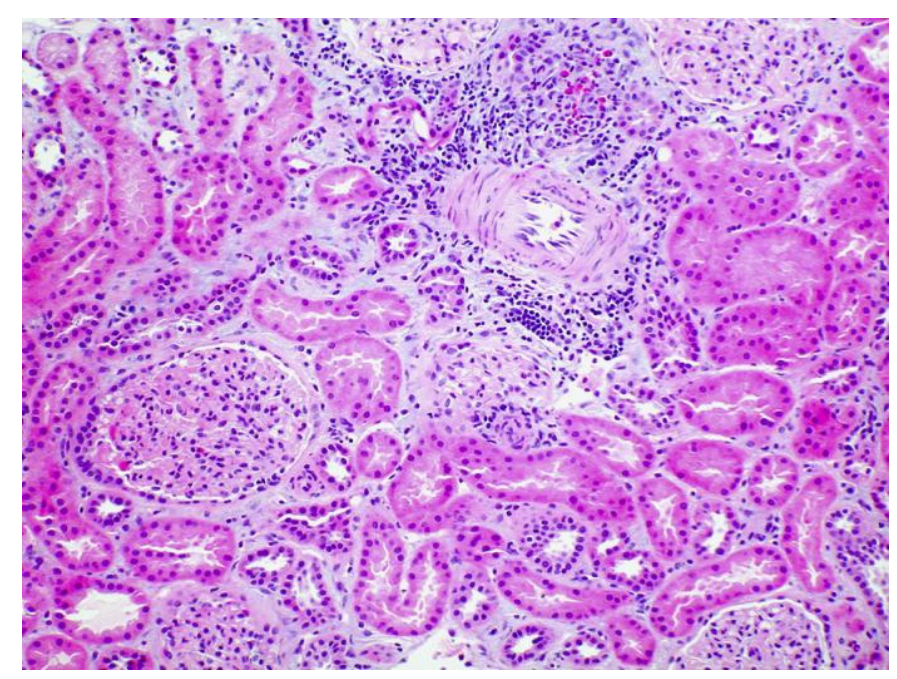

Fig. 2. TINU. Mature lymphocytes and few eosinophils are noted in the mildly edematous interstitium; glomeruli are normal. 